\title{
Special notice to readers and authors on scientific misconduct
}

\author{
Donald R. Miller, MD
}

Published online: 28 March 2009

(C) Canadian Anesthesiologists' Society 2009

We were informed earlier in 2009 that Baystate Medical Center (Baystate) at Tufts University in Boston had conducted an investigation, pursuant to their Policy on Misconduct in Research and Scholarly Activities, regarding a number of research studies conducted by Dr. Scott Reuben. These proceedings were part of the Baystate Medical Review Process, which is confidential and privileged under Massachusetts law. The Baystate investigation determined that, "Dr. Reuben fabricated data reported in the referenced articles and that all fabricated data were created under the sole discretion of Dr. Reuben." The complete list of articles (Appendix) spans a period of 12 years of scientific investigation from 1996 to 2008. A series of retraction notices has been issued in the affected journals. Drs. Eisenach, ${ }^{1}$ Shafer, ${ }^{2}$ and White et al. have written several excellent editorials highlighting the broader implications regarding the unimpeached literature and the damage to the body of scientific knowledge resulting from this extensive data fabrication and article retraction. ${ }^{3}$

The purpose of this notice is two-fold. First, we alert our readers to the fact that Dr. Scott Reuben has previously published two articles in the Journal. ${ }^{4,5}$ The first article, published in 2005, is a report of an original investigation, the second article is a meeting abstract published in 2006. The areas of clinical investigation in these two articles are similar to those in articles other journals have retracted. This past January, our communication with Baystate Medical Center regarding the two articles published in the Journal revealed only that these articles had not been

D. R. Miller, MD $(\bowtie)$

Editorial Office, Canadian Journal of Anesthesia, c/o Department of Anesthesia, The Ottawa Hospital, General Campus, CCW Room 1409, 501 Smyth Road, Ottawa, ON K1H 8L6, Canada e-mail: dmiller@ottawahospital.on.ca identified by their ongoing investigation of scientific misconduct. However, while we will attempt to seek further clarification, we have no basis at the present time to either retract or suppress the articles published by Dr. Reuben in the Journal. This position does not preclude our advising readers of the retraction of Dr. Reuben's articles from other journals, as there is overlap regarding both the area of scientific investigation and the publication timeframe.

Second, in keeping with the approach adopted by other journals, we advise authors submitting articles to the Journal that we will maintain a list of Dr. Reuben's retracted articles to ensure that not one of them is cited in new manuscripts advancing through our peer review system. Unfortunately, we recognize that authors may continue to cite work that has been discredited, even after it has been exposed as fraudulent. ${ }^{6}$ For instance, this could occur if an author were to cite an article from the printed copy of a journal without being aware that the article had been retracted. In general, authors should check their references online rather than rely on the print version. We also recognize the possibility that journals may fail to retract an article despite having received notice of scientific misconduct. ${ }^{7}$ As highlighted in a study by Budd et al., the volume of citations following article retraction can be substantial. During their study, they reviewed 235 biomedical articles which had been retracted, and they found that the articles had been cited 2034 times after retraction notices were issued. ${ }^{8}$ Disturbingly, it was found that many authors who had cited the retracted articles treated those citations as valid research. This observation challenges authors to ensure that cited articles are interpreted accurately.

Authors, editors, and editorial boards have a duty to ensure the integrity of the scientific literature, as do research ethics boards and their institutions, study sponsors, and 
research mentors. A broader discussion of the issue of scientific misconduct in the medical literature is beyond the scope of this notice to our readers. However, we advise readers and authors that editors of anesthesia journals communicate regularly on a variety of matters of mutual interest, including manuscripts which raise concern ${ }^{9}$ of scientific misconduct that transcends individual journals. As mentioned above, we will maintain up-to-date lists of retracted articles to ensure that none is cited in future publications of the Journal. Finally, our editorial board will continue to review and update editorial policies and procedures to maintain consistency with the "Guidelines on Good Publication Practice" published by The Committee on Publication Ethics (www.publicationethics.org), of which the Journal is a member.

\section{Avis spécial à l'intention des lecteurs et des auteurs concernant la fraude scientifique}

Plus tôt en 2009, nous avons été informés que le Centre médical de Baystate (Baystate) de l'université Tufts à Boston menait une enquête conformément à leur politique sur la fraude scientifique dans les activités de recherche et d'érudition concernant plusieurs études menées par le Dr Scott Reuben. Ces investigations s'inscrivaient dans le processus de révision médicale de Baystate, lequel est confidentiel et couvert par le secret professionnel selon les lois du Massachusetts. L'enquête de Baystate a conclu que « le Dr Reuben a fabriqué des données rapportées dans les articles cités en référence et toutes les données fabriquées ont été créées à la seule discrétion du Dr Reuben. » La liste complète des articles (voir annexe) couvre une période de 12 ans de recherches scientifiques allant de 1996 à 2008. Une série d'avis de rétractation a été publié dans les revues concernées. Les Drs Eisenach, ${ }^{1}$ Shafer, ${ }^{2}$ et White et coll. ont écrit plusieurs excellents éditoriaux qui soulignent les implications plus larges d'une littérature irréprochable et les dommages au corpus de connaissances scientifiques qui résultent d'une si vaste fabrication de données et de la rétractation d'articles. ${ }^{3}$

L'objectif de cet avis est double. En premier lieu, nous tenons à informer nos lecteurs que le Dr Scott Reuben a publié deux articles dans le Journal par le passé. ${ }^{4,5}$ Le premier article, publié en 2005, est un compte-rendu de recherche originale, et le second est un résumé d'un congrès publié en 2006. Les domaines de recherche clinique de ces deux articles sont semblables à ceux des articles qui ont été rétractés par d'autres revues. Au mois de janvier dernier, notre correspondance avec le Centre médical de
Baystate concernant les deux articles publiés dans le Journal a seulement révélé que ces articles n'avaient pas été identifiés dans leur enquête en cours sur la fraude scientifique. Toutefois, tandis que nous nous employons à obtenir des clarifications, nous n'avons à l'heure actuelle aucun fondement sur lequel nous appuyer pour rétracter ou supprimer les articles publiés par Dr Reuben dans le Journal. Cette situation ne nous empêche cependant pas d'avertir les lecteurs que des articles du Dr Reuben ont été rétractés d'autres revues, étant donné qu'il y a chevauchement aussi bien au niveau du domaine de recherche scientifique que des dates de publication.

Deuxièmement, dans la lignée de l'approche adoptée par d'autres revues, nous avertissons les auteurs soumettant des articles au Journal que nous conserverons une liste des articles rétractés du Dr Reuben afin de garantir qu'aucun n'est cité dans les nouveaux manuscrits qui sont soumis pour publication et sont dans notre système d'évaluation par les pairs. Malheureusement, nous sommes conscients que les auteurs peuvent continuer à citer des travaux qui ont été discrédités et ce, même après que la fraude a été dévoilée. ${ }^{6}$ Par exemple, une telle situation pourrait survenir si un auteur citait un article de la version imprimée d'une revue sans savoir que l'article a été rétracté. De manière générale, les auteurs devraient vérifier leurs références en ligne plutôt que de s'appuyer uniquement sur la version imprimée. Nous sommes également conscients qu'il existe une possibilité que certaines revues ne rétractent pas un article malgré avoir été informées de la fraude scientifique. ${ }^{7}$ Comme cela a été souligné dans une étude de Budd et coll., le nombre de citations après la rétractation d'un article peut être considérable. Au cours de leur étude, Budd et coll. ont passé en revue 235 articles biomédicaux qui avaient été rétractés, et ils ont découvert que les articles avaient été cités 2034 fois après la publication des avis de rétractation. ${ }^{8}$ Ce qui est troublant, c'est que bon nombre d'auteurs ayant cité les articles rétractés traitaient ces citations comme des références valables. Cette observation met les auteurs au défi de garantir que les articles qu'ils citent sont interprétés de façon précise.

Il est du devoir des auteurs, des réviseurs et des bureaux de rédaction de garantir l'intégrité de la littérature scientifique, tout comme le font les comités d'éthique de la recherche et leurs institutions, les commanditaires et les mentors de recherche. Une discussion plus large de la question de fraude scientifique dans la littérature médicale va bien au-delà de l'intention de cet avis à nos lecteurs. Cependant, nous tenons à informer les lecteurs et les auteurs que les rédacteurs en chef des revues d'anesthésie se consultent régulièrement sur toutes sortes de questions d'intérêt commun, y compris sur les manuscrits qui soulèvent des inquiétudes ${ }^{9}$ quant à la fraude scientifique, des questions qui transcendent chacune des revues. Comme nous l'avons mentionné ci-dessus, nous conserverons des 
listes mises à jour des articles rétractés afin de garantir qu'aucun n'est cité dans les publications futures du Journal. Enfin, notre bureau de rédaction continuera de réviser et de mettre à jour nos politiques et procédures éditoriales pour assurer qu'elles sont conformes aux « Directives sur la bonne pratique de publication » (Guidelines on Good Publication Practice) publiées par le Comité sur l'éthique de publication (The Committee on Publication Ethics) (www.publicationethics.org), duquel le Journal fait partie.

\section{Appendix: Retracted articles}

Reuben SS, Connelly NR. Postarthroscopic meniscus repair analgesia with intraarticular ketorolac or morphine. Anesth Analg 1996; 82: 1036-9.

Reuben SS, Connelly NR, Maciolek H. Postoperative analgesia with controlled-release oxycodone for outpatient anterior cruciate ligament surgery. Anesth Analg 1999; 88: 1286-91.

Reuben SS, Reuben JP. Brachial plexus anesthesia with verapamil and/or morphine. Anesth Analg 2000; 91: 379-83.

Reuben SS, Connelly NR. Postoperative analgesic effects of celecoxib or rofecoxib after spinal fusion surgery. Anesth Analg 2000; 91: 1221-5.

Reuben SS, Vieira P, Faruqui S, Verghis A, Kilaru P, Maciolek $H$. Local administration of morphine to bone following spinal fusion surgery. Anesthesiology 2001; 95 : 390-4.

Reuben SS, Fingeroth $R$, Krushell $R$, Maciolek $H$. Evaluation of the safety and efficacy of the perioperative administration of rofecoxib for total knee arthroplasty. J Arthroplasty 2002; 17: 26-31.

Reuben SS, Steinberg RB, Maciolek H, Manikantan P. An evaluation of the analgesic efficacy of intravenous regional anesthesia with lidocaine and ketorolac using a forearm versus upper arm tourniquet. Anesth Analg 2002; 95: 457-60.

Reuben SS, Gutta SB, Sklar J, Maciolek H. Effect of initiating a multimodal analgesic regimen upon patient outcomes after anterior cruciate ligament reconstruction for same-day surgery: a 1200-patient case series. Acute Pain 2004; 6: 87-93.

Reuben SS, Rosenthal EA, Steinberg RB, Faruqi S, Kilaru $P R$. Surgery on the affected upper extremity of patients with a history of complex regional pain syndrome: the use of intravenous regional anesthesia with clonidine. J Clin Anesth 2004; 16: 517-522.

Reuben SS, Makari-Judson G, Lurie SD. Evaluation of efficacy of the perioperative administration of venlafaxine $\mathrm{XR}$ in the prevention of postmastectomy pain syndrome. J Pain Symptom Manage 2004; 27: 133-9.
Reuben $S$. The effect of intraoperative valdecoxib administration on PGE2 levels in the CSF. J Pain 6 Suppl 1: S21 (Abstract 649).

Reuben SS, Ekman EF. The effect of cyclooxygenase-2 inhibition on analgesia and spinal fusion. J Bone Joint Surg Am 2005; 87: 536-42.

Reuben SS, Gutta SB, Maciolek H, Sklar J, Redford R. Effect of initiating a preventative multimodal analgesic regimen upon long-term patient outcomes after anterior cruciate ligament reconstruction for same-day surgery: a 1200-patient case series. Acute Pain 2005; 7: 65-73.

Reuben SS, Pristas R, Dixon D, Faruqi S, Madabhushi $L$, Wenner $S$. The incidence of complex regional pain syndrome after fasciectomy for Dupuytren's contracture: a prospective observational study of four anesthetic techniques. Anesth Analg 2006; 102: 499-503.

Reuben SS, Buvanendran A, Kroin JS, Raghunathan K. The analgesic efficacy of celecoxib, pregabalin, and their combination for spinal fusion surgery. Anesth Analg 2006; 103: 1271-7.

Reuben SS, Buvenandran A, Kroin JS, Raghunathan K. Analgesic efficacy of celecoxib, pregabalin, and their combination for spinal fusion surgery. Anesthesiology 2006; 105: A1194.

Reuben SS, Buvanendran A, Kroin JS, Steinberg RB. Postoperative modulation of central nervous system prostaglandin E2 by cyclooxygenase inhibitors after vascular surgery. Anesthesiology 2006; 104: 411-6.

Reuben SS, Ekman EF, Raghunathan K, Steinberg RB, Blinder JL, Adesioye $J$. The effect of cyclooxygenase-2 inhibition on acute and chronic donor-site pain after spinalfusion surgery. Reg Anesth Pain Med 2006; 31: 6-13.

Reuben SS, Ekman EF, Charron D. Evaluating the analgesic efficacy of administering celecoxib as a component of multimodal analgesia for outpatient anterior cruciate ligament reconstruction surgery. Anesth Analg 2007; 105: 222-7.

Reuben SS, Ekman EF. The effect of initiating a preventive multimodal analgesic regimen on long-term patient outcomes for outpatient anterior cruciate ligament reconstruction surgery. Anesth Analg 2007; 105: 228-32.

Reuben SS, Buvenandran A, Katz B, Kroin JS. A prospective randomized trial on the role of perioperative celecoxib administration for total knee arthroplasty: improving clinical outcomes. Anesth Analg 2008; 106: 1258-64.

\section{References}

1. Eisenach J. Data fabrication and how not to get lost in the woods. Anesthesiology 2009; doi:10.1097/ALN.0b013e3181a066f9.

2. Shafer $S$. Tattered threads. Anesth Analg 2009; doi:10.1213/ANE. 0b013e3181a16846. 
3. White PF, Kehlet H, Liu S. Perioperative analgesia-what do we still know? Anesth Analg 2009; doi:10.1213/ANE.0b013e318 $1 \mathrm{a} 16835$.

4. Reuben SS, Ablett D, Kaye R. High dose nonsteroidal antiinflammatory drugs compromise spinal fusion. Can J Anesth 2005; 52: 506-12.

5. Kuppinger JG, Blinder JL, Reuben SS. Valdecoxib pharmacokinetics in human cerebrospinal fluid. Can J Anesth 2006; 52: A161 (abstract).
6. Couzin J, Unger K. Scientific misconduct. Cleaning up the paper trail. Science 2006; 312: 38-43.

7. Sox HC, Rennie D. Research misconduct, retraction, and cleansing the medical literature: lessons from the Poehlman case. Ann Intern Med 2006; 144: 609-13.

8. Budd JM, Sievert ME, Schultz TR. Phenomena of retraction: reasons for retraction and citations to the publications. JAMA 1998; 280: 296-7.

9. Todd MM. A lesson learned. Can J Anesth 2005; 52: 770-1. 